\title{
COMPRESSIBLE GAS FLOW THROUGH THE DIFFUSIBLE BARRIERS AND THE POROUS MEDIA
}

\author{
M. Kyncl ${ }^{*}$, J. Pelant ${ }^{* *}$
}

\begin{abstract}
This paper is focused on the numerical simulation of the compressible gas flow through the porous media and through the diffusible barriers. We work with the the non-stationary viscous compressible fluid flow, described by the RANS equations. The flow through the porous media is characterized by the loss of momentum. For the simulation of the diffusible barrier we analyze the modification of the Riemann problem with one-side initial condition, complemented with the Darcy's law and added inertial loss. The presented examples were obtained with the own-developed code for the solution of the compressible gas flow.
\end{abstract}

Keywords: compressible gas flow, diffusible barriers, the RANS equations, the Riemann problem, boundary conditions

\section{Introduction}

We work with the compressible fluid motion described by the conservation laws of mass, momentum, and energy. These fundamental conservation laws form a system of partial differential equations (the Euler equations, the Navier-Stokes equations, the Navier Stokes equations with turbulent models). We choose the well-known finite volume method to discretize the analytical problem, represented by the system of the equations in generalized (integral) form. We split the area of the interest into the elements, and we construct a piecewise constant solution in time. The crucial problem of this method lies in the evaluation of the so-called fluxes through the faces of the elements. Here we use the analysis of the Riemann problem to construct these fluxes. The analysis of the Riemann problem with original modifications is used at the boundary (see Kyncl (2011); Kyncl and Pelant (2014, 2016)), and for the simulation of the diffusible barrier, presented also in Kyncl and Pelant (2013), and further developed in Kyncl and Pelant (2017). Here we show own algorithm for the solution of the boundary problem at the diffusible barrier, and we use it in the numerical examples.

\section{Equations}

The system of conservation laws can be written in the following vector form

$$
\frac{\partial \boldsymbol{w}}{\partial t}+\sum_{s=1}^{3} \frac{\partial \boldsymbol{f}_{s}(\boldsymbol{w})}{\partial x_{s}}=\sum_{s=1}^{3} \frac{\partial \boldsymbol{R}_{s}(\boldsymbol{w}, \nabla \boldsymbol{w})}{\partial x_{s}}+\boldsymbol{S}(\boldsymbol{w}) \quad \text { in } Q_{T}=\Omega \times(0, T) .
$$

Here $\boldsymbol{w}=\boldsymbol{w}(x, t)$ is the state vector, $x \in \Omega, t$ denotes the time, $Q_{T}$ is the space-time cylinder, $\boldsymbol{f}_{s}$ are the inviscid fluxes, $\boldsymbol{R}_{s}$ are the viscous fluxes, $\boldsymbol{S}$ is the source-term vector. Further we use the equation of state of ideal gas, and the turbulent model equations.

\section{Porous media simulation}

The porous media is simulated using the modification of the system of equations (1). The simple porous media can be simulated via the new source term, written as 


$$
\boldsymbol{S}=\left(\begin{array}{c}
0 \\
-\frac{\mu}{\alpha} v_{1}-C_{0} \frac{\varrho}{2}|\boldsymbol{v}| v_{1}+\varrho g_{1} \\
-\frac{\mu}{\alpha} v_{2}-C_{0} \frac{\varrho}{2}|\boldsymbol{v}| v_{2}+\varrho g_{2} \\
-\frac{\mu}{\alpha} v_{3}-C_{0} \frac{\varrho}{2}|\boldsymbol{v}| v_{3}+\varrho g_{3} \\
-\frac{\mu}{\alpha} \boldsymbol{v}^{2}-C_{0} \frac{\varrho}{2}|\boldsymbol{v}| \boldsymbol{v}^{2}+\varrho \boldsymbol{g} \cdot \boldsymbol{v}
\end{array}\right)
$$

Here $\alpha$ is the permeability coefficient, $C_{o}$ is the pressure gradient coefficient, $\varrho$ is the density, $\boldsymbol{v}=$ $\left(v_{1}, v_{2}, v_{3}\right)$ is the velocity vector, $\boldsymbol{g}=\left(g_{1}, g_{2}, g_{3}\right)$ is the gravitational acceleration vector, $\mu$ denotes the dynamic viscosity. The source term (2) was implemented into own-developed software, Figure 1 shows computed example.
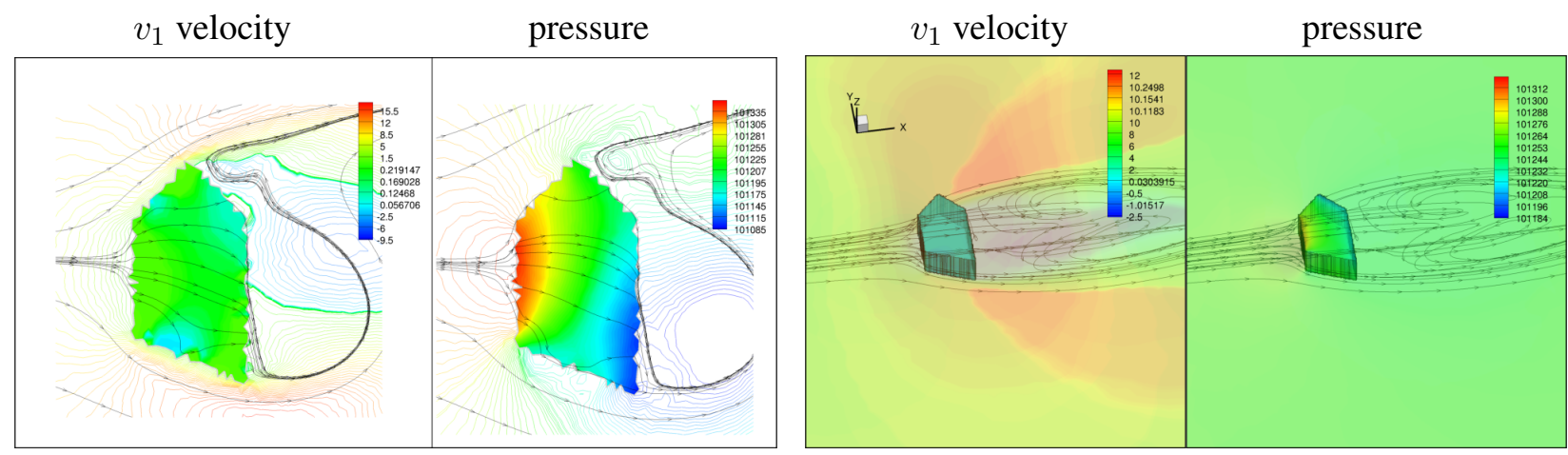

Fig. 1: The compressible gas flow through the polygonal porous area, isolines of $v_{1}$ velocity component and the pressure, computational results for the regime $10\left[\mathrm{~m} \mathrm{~s}^{-1}\right], 2 D$ inviscid simulation, and $3 D$ viscous turbulent simulation in the gravitational field.

\section{Boundary condition for the diffusible barrier}

Here we present the diffusible barrier condition as a combination of Darcy's law with the additional innertial losses.

$$
\frac{\Delta p}{\Delta m}=-\left(\frac{\mu}{\alpha} U+C_{o} \frac{1}{2} R U^{2}\right)
$$

where $\Delta p=p_{2}-p_{1}$ is the pressure difference across the barrier, $\Delta m$ is the thickness of the barrier (example $\Delta m \in(0.01,0.05)), \mu$ is the dynamic viscosity, $\alpha$ the permeability coefficient (example $\alpha=10^{-8}$ ), $C_{o}$ is the pressure gradient coefficient (example $C_{o}=10^{2}$ ), $R$ the density at the barrier, and $U$ is the velocity at the barrier. The equation (3) can be rewritten in the form

$$
R U^{2}+C U=d\left(p_{1}-p_{2}\right),
$$

here $C=\frac{2 \mu}{\alpha C_{o}}>0, d=\frac{2}{\Delta m C_{o}}>0,\left(p_{1}-p_{2}\right) \geq 0$. We are interested in the boundary values $R, U, P$ at the barrier. Further we require that the conservation laws (1D Euler equations) are satisfied in the close vicinity of the barrier

$$
\frac{\partial}{\partial t}\left(\begin{array}{c}
\varrho \\
\varrho u \\
E
\end{array}\right)+\frac{\partial}{\partial \tilde{x}_{1}}\left(\begin{array}{c}
\varrho u \\
\varrho u^{2}+p \\
(E+p) u
\end{array}\right)=0, \quad t \in(0, \infty), \tilde{x}_{1} \in(-\infty, \infty) .
$$

Here the axis $\tilde{x}_{1}$ is perpendicular to the barrier, $\varrho\left(\tilde{x}_{1}, t\right)$ denotes the density, $p\left(\tilde{x}_{1}, t\right)$ is the pressure, $u\left(\tilde{x}_{1}, t\right)$ is the velocity (with the direction perpendicular to the barrier), $E\left(\tilde{x}_{1}, t\right)$ denotes the total energy: $E=$ $\varrho u^{2} / 2+p /(\gamma-1)$. The initial condition is formed by the two states near the barrier. Let us denote these states $\varrho_{1}, u_{1}, p_{1}$ (for $\tilde{x}_{1}<0$ ) and $\varrho_{2}, u_{2}, p_{2}$ (for $\tilde{x}_{1}>0$ ). Further we think of the barrier problem as of two boundary problems (for the inlet, and for the outlet) with the two particular solutions (different in general). The analysis of these problems was shown in Kyncl and Pelant (2017), Figures 2, 3 show the resulting algorithm. We suppose the initial velocity $u_{1} \geq 0$, and we seek the solution with $U>0$. 
INLET to the diffusible barrier

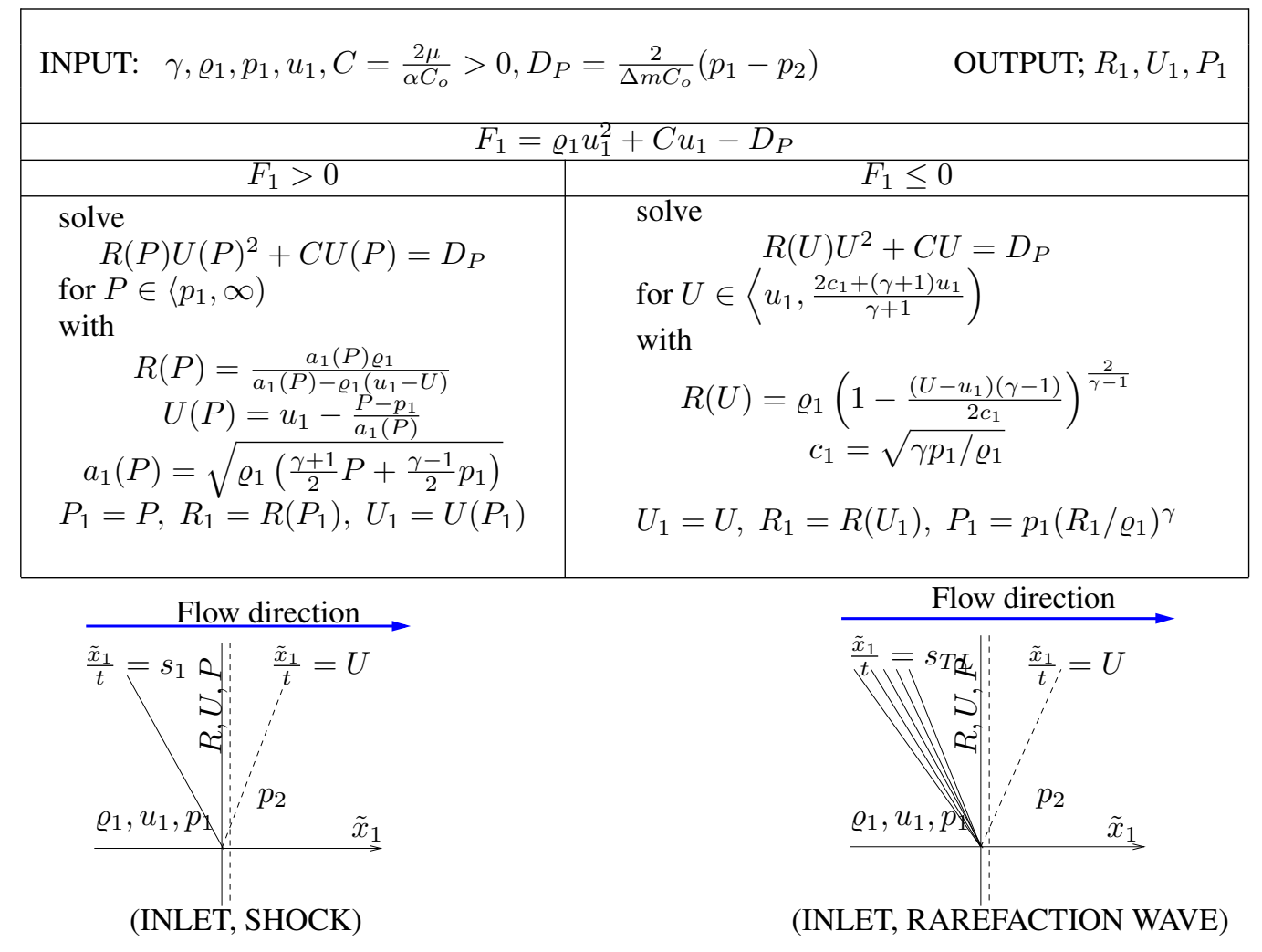

Fig. 2: Algorithm for the solution of the Problem 1 (INLET to the barrier). Both posibilities are taken into account.

OUTLET from the diffusible barrier

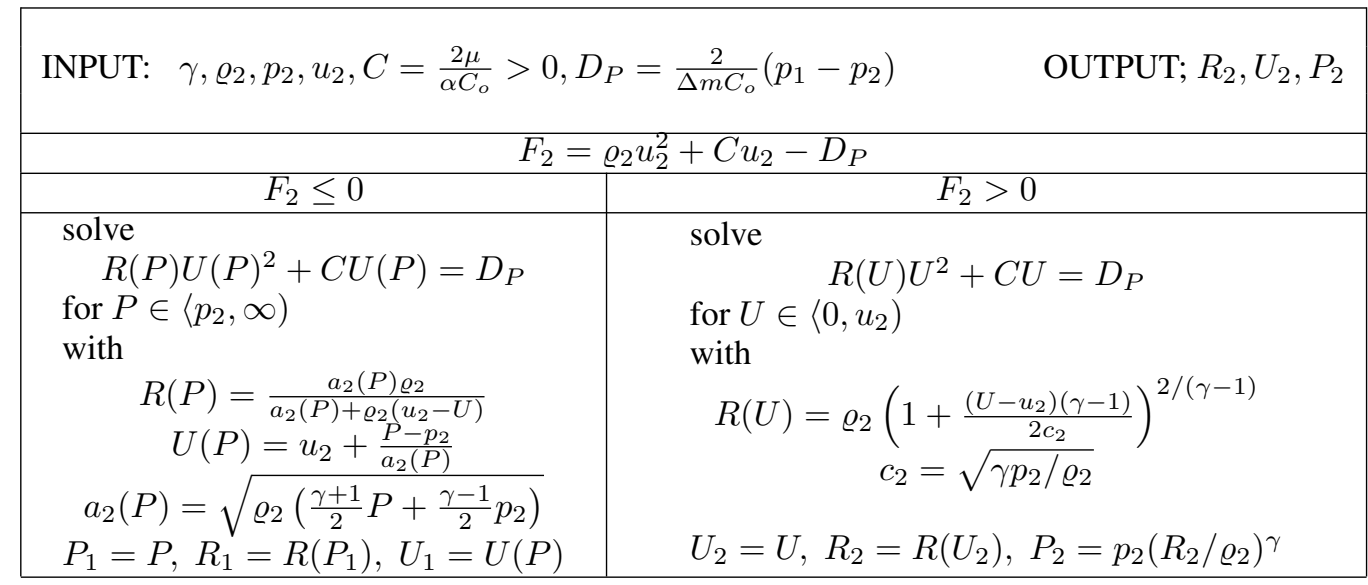

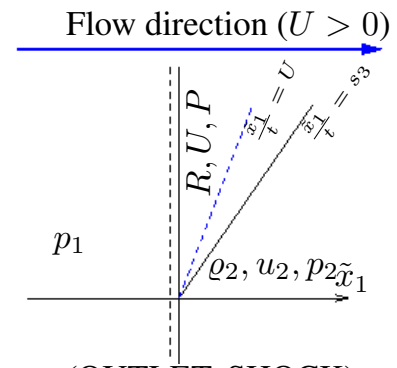

(OUTLET, SHOCK)

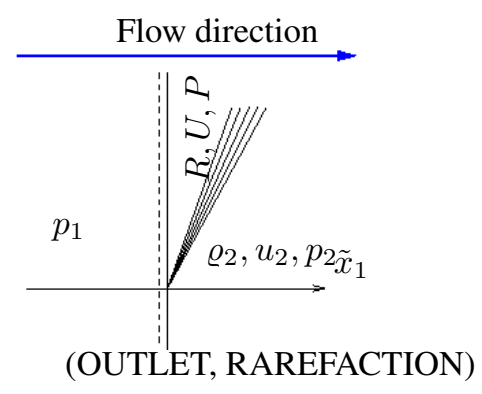

Fig. 3: Algorithm for the solution of the Problem 2 (OUTLET from the barrier). Both posibilities are taken into account. 


\section{Examples}

The presented algorithm was implemented into the own-developed code, and used on the numerical examples. The Figures 4, 5 show the visual comparison of the flow through the diffusible barrier composed of multiple rigid plates, porous media, and the diffusible barrier. The velocity profiles at chosen vertical cuts are presented.
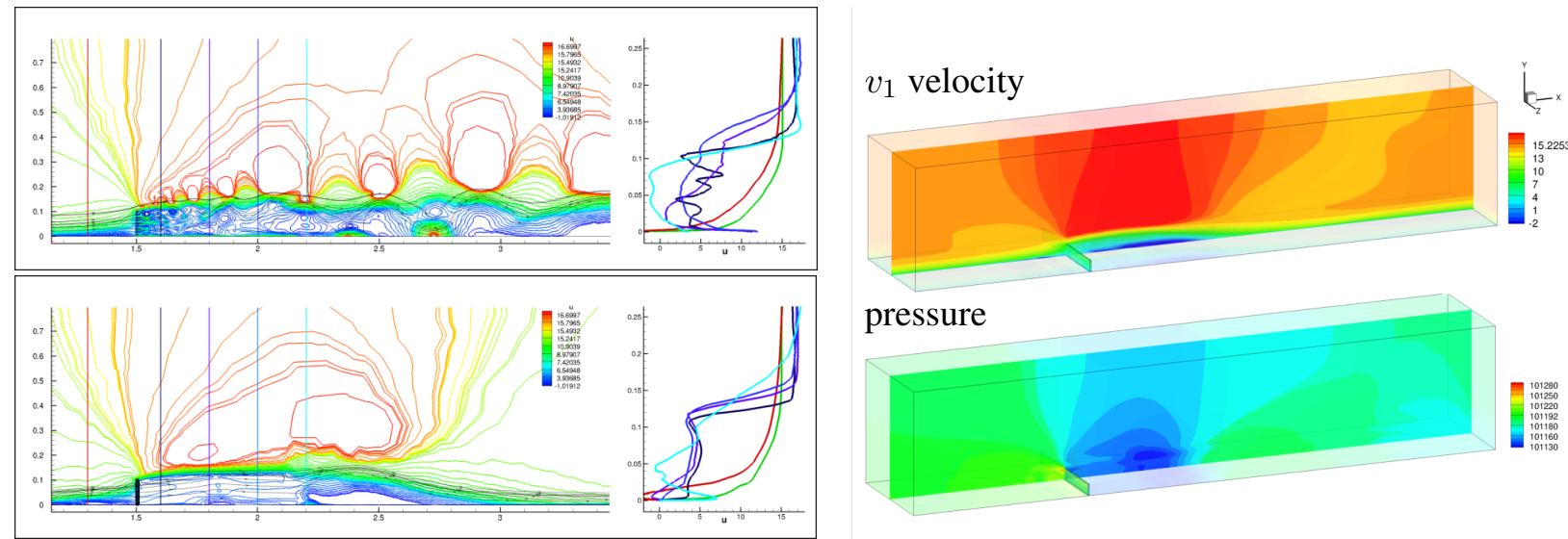

\section{pressure}

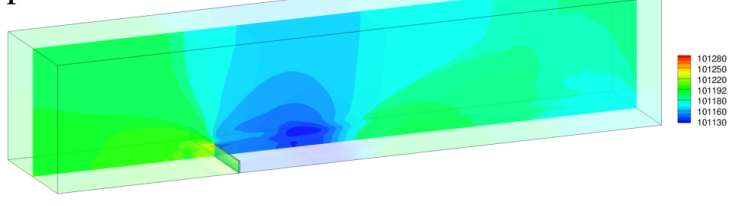

Fig. 4: Numerical simulation of the barrier composed of multiple plates, comparison with porous media, and $3 D$ simulation. Regime $15\left[\mathrm{~m} \mathrm{~s}^{-1}\right]$, barrier height $h=0.1[\mathrm{~m}]$, results at the time instant $t=0.2[\mathrm{~s}]$.

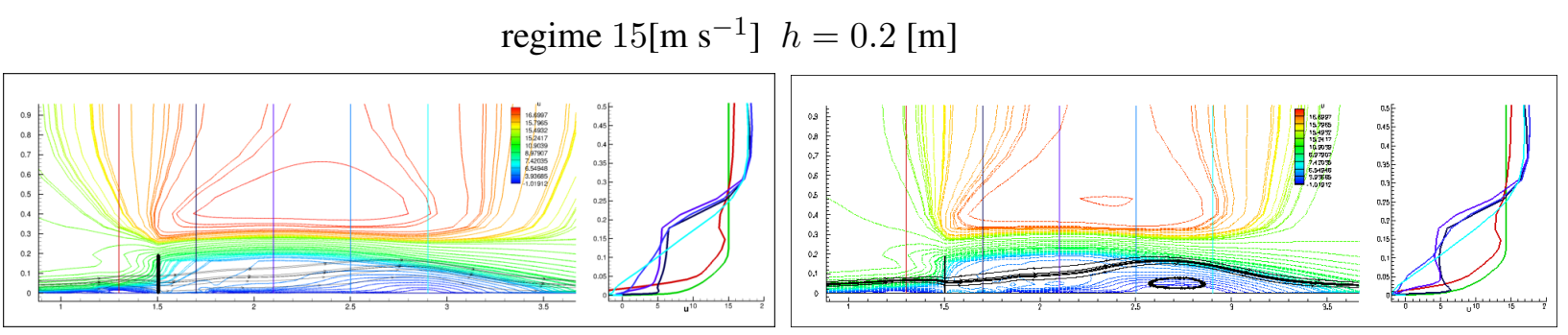

Fig. 5: Numerical simulation of the barrier simulated as porous media (left), and diffusible barrier (right).

\section{Conclusions}

The paper works with the compressible viscous gas flow, with the focus on the porous media and the diffusible barrier. The original boundary condition for the diffusible barrier (analyzed by authors also in Kyncl and Pelant $(2013,2017))$ is presented, together with the computational algorithm. It is based on the analysis of the Riemann problem for the split Euler equations and the modifications of this problem. Here the left hand side initial condition is replaced by given complementary conditions. The resulting algorithm was implemented into the own-developed software, and used in numerical examples.

\section{Acknowledgments}

The results originated with the support of Ministry of Industry and Trade of the Czech Republic for the long-term strategic development of the research organization. The authors acknowledge this support.

\section{References}

Kyncl M. (2011), Numerical solution of the three-dimensional compressible flow, Doctoral Thesis, Prague, 2011.

Kyncl M. and Pelant J. (2013), Numerical simulation of the flow around diffusible barriers, EPJ WoC, Vol. 45, 2013, EFM12, 01054.

Kyncl M. and Pelant J. (2014), The Initial-Boundary Riemann Problem for the Solution of the Compressible Gas Flow, ECCOMAS 2014 Proceedings.

Kyncl M. and Pelant J. (2016), Modification of the Riemann problem and the application for the boundary conditions in computational fluid dynamics, EPJ WoC, Vol. 143, 2017, EFM16, 02061.

Kyncl M. and Pelant J. (2017), The simulation of the flow through the porous media and diffusible barriers, EFM 2017 Proceedings. 